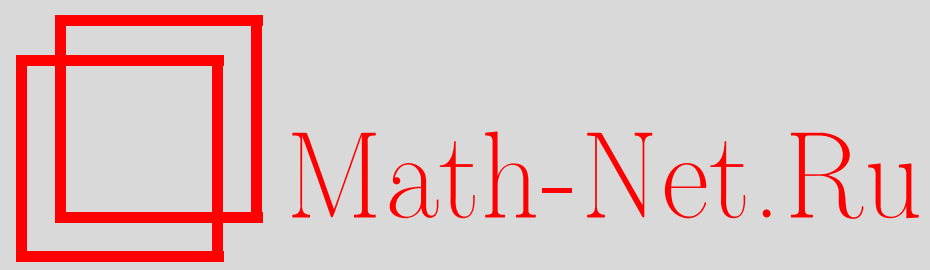

В. К. Булитко, О рекурсивно сжимаемых множествах, $M a$ тем. заметки, 1998, том 64, выпуск 1, 9-16

DOI: https://doi.org/10.4213/mzm1367

Использование Общероссийского математического портала Math-Net.Ru подразумевает, что вы прочитали и согласны с пользовательским соглашением http://www.mathnet.ru/rus/agreement

Параметры загрузки:

IP : 54.210 .77 .194

26 апреля 2023 г., 16:22:51

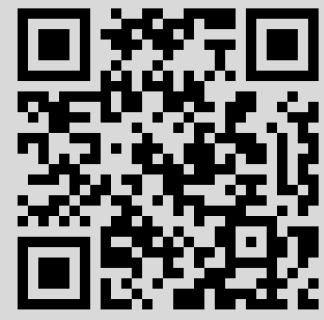




\title{
О РЕКУРСИВНО СЖИМАЕМЫХ МНОЖЕСТВАХ
}

\author{
В.К. Булитко
}

\begin{abstract}
Изучаются классы рекурсивно сжимаемых и несжимаемых множеств, как и некоторые другие классы, возникающие в связи с одной простейшей теоретико-рекурсивной моделью упаковки массивов данных. Получены некоторые новые критерии полноты множеств.

Библиография: 8 названий.
\end{abstract}

Проблема упаковки данных часто возникает в практике информационных преобразований. Целью является сжатие объема массива данных при сохранении возможности эффективного восстановления исходного массива $A$ по сжатому массиву $B$.

В настоящей заметке мы рассмотрим некоторые результаты, вытекающие из следующей простейшей модели, не имеющей, впрочем, практического значения. Рассматриваем только одномерные бесконечные массивы $A[0: \infty]$, состоящие из 0 и 1 , т.е. $A[i] \in\{0,1\}, i \in \mathbb{N}$. Такой массив $A$, очевидно, можно представить множеством, для которого $A$ является характеристической функцией. Модель, которую мы имеем ввиду, соответствует следующему условию: если массив $B$ является более плотной упаковкой массива $A$, то множество $A$ можно свести по Тьюрингу к множеству $B$, причем начальный сегмент множества $A$ длины $l, l \in \mathbb{N}$, восстанавливается по более короткому начальному сегменту множества $B$. Для уточнения этого условия далее мы будем рассматривать множества и использовать понятие относительной рекурсивности с ограниченньм доступом к оракулу [1] (однако мы не предполагаем знакомства с этой работой). Кроме того, здесь мы не будем касаться вопросов существования и качества алгоритмов упаковки-сжатия, а также вопросов качества алгоритмов распаковки.

Рассматриваемая модель близка по духу к моделям сжатия информации, опирающимся на понятие сложности разрешения KR (Марков, Ловеланд). Теорема 2 ниже является по существу модификацией известной теоремы Барздиня. А первое предложение теоремы 4 аналогично хорошо известному результату о том, что алгоритмически случайные последовательности образуют множество полной меры в обычном метрическом пространстве бесконечных 01-последовательностей.

В работе [2] Соловьёв рассматривает сводимость конечными автоматами бесконечных 01-последовательностей. Это приводит к иным уточнениям понятия сжимаемости и иным результатам. Однако, прослеживаются и некоторые параллели. Например, понятию жесткой последовательности из [2] у нас соответствует понятие рекурсивно не сжимаемого множества, а понятие плотно упакованной последовательности может быть 
в некоторой мере представлено процедурой нетривиального сжатия множества с сохранением его степени неразрешимости. Конечно, эти аналогии достаточно отдаленные, поскольку, с одной стороны, Соловьёв рассматривает чрезвычайно сильную с точки зрения теории рекурсии сводимость. С другой стороны, в [2] сводящий конечньй автомат $\mathfrak{A}$ при построении начального сегмента длины $n$ множества $A$, сводимого им к множеству $B$, может использовать информацию о начальном сегменте длины $n+c$ множества $B$ для некоторой константы $c$, определяемой автоматом $\mathfrak{A}$.

Неопределяемые понятия и обозначения взяты из [3]. Пусть $\mathscr{T}$ обозначает класс всех тотальных функций $f: \mathbb{N} \rightarrow \mathbb{N}$, а $\mathscr{T}^{+}$состоит из всех неубьвающих функций $f \in \mathscr{T}$ с бесконечным Val $f ; I$ - тождественная функция; $A^{\sigma}$ есть множество $A$, если $\sigma=1$, и $\bar{A}$ в противном случае. Если $B$ - конечное непустое множество, то $\max (B)(\min (B))$ обозначает максимальньй (минимальньй) элемент $B ; \max (\varnothing)=\min (\varnothing)=0$. Запись $A \leqslant_{f} B$ обозначает существование $z, x_{0} \in \mathbb{N}$ таких, что $A \leqslant_{T}^{z} B$ и $x \in A^{\sigma} \Longleftrightarrow(\exists u, v)$ $\left(\langle x, \sigma, u, v\rangle \in W_{z} \& D_{u} \subseteq B \& D_{v} \subseteq \bar{B} \&\left(x_{0} \leqslant x \Longrightarrow \max \left(D_{u} \cup D_{v}\right) \leqslant f(y)\right)\right)$ для каждых $\sigma \in\{0,1\}$ и $x, y$, удовлетворяюших $x \leqslant y$;

$$
x-y= \begin{cases}x-y, & \text { если } y \leqslant x, \\ 0 & \text { иначе }\end{cases}
$$

$|A|$ обозначает мошность множества $A$. Наконец, $\|w\|$ обозначает длину слова $w$, и если $w$ - последовательность $w(0) w(1) \ldots w(n) \ldots$, то $\left.w\right|_{m} ^{n} \stackrel{\mathrm{df}}{=} w(m) w(m+1) \ldots w(n)$, где $m \leqslant n$. Характеристическую функцию произвольного множества $A$ обозначаем через $\mathscr{C}_{A}$ и рассматриваем как бесконечную 01-последовательность $\mathscr{C}_{A}(0) \mathscr{C}_{A}(1) \mathscr{C}_{A}(2) \ldots$ Всякий начальньй отрезок $\left.\mathscr{C}_{A}\right|_{0} ^{n}$ этой последовательности назьваем сегментом.

Ясно, что $A$ - рекурсивное множество, если

$$
A \leqslant_{f} B \& \underset{n \rightarrow \infty}{\lim _{n \rightarrow \infty}} f(n)<\infty
$$

Пусть $\Gamma \subseteq \mathscr{T}^{+}$. Мы скажем, что $A$ есть $\Gamma$ - сжимаемое, если существует функция $h \in \Gamma$ такая, что $(\exists B \subset \mathbb{N})(A \leqslant(I-h) B)$, иначе множество $A$ есть $Г$-несжимаемое. Рекурсивно сжимаемым множеством (РСМ) назьвается Г-сжимаемое множество, когда Г есть множество всех неубьвающих общерекурсивных функций с бесконечным множеством значений.

ЛЕмма 1. (A есть Г-сжимаемое множество $) \Longleftrightarrow(\bar{A}$ есть Г-сжимаемое множсество).

Доказательство очевидно.

Лемма 2. Пусть $R$-рекурсивное мнохество, $R \cap A$ бесконечно, $u(\exists X \subseteq R)$ $\exists z\left(A \cap R \leqslant_{(I-h)}^{z} X\right)$, әде $h \in \mathscr{T}^{+}$рекурсивная функиия, $и$

$$
\varlimsup_{n \rightarrow \infty}|R \cap\{h(n), \ldots, n\}|=\infty
$$

Тогда A есть PCM. 
ДокАЗАТЕЛЬство. Если $\bar{R}$ конечно, то доказьвать нечего.

Будем обозначать $i$-й в порядке возрастания элемент произвольного бесконечного множества $B$ через $B(i)$. Пусть $q^{\prime}$ будет рекурсивной функцией, удовлетворяющей следуюшим условиям:

1) $\left|\left\{h\left(q^{\prime}(n)\right), \ldots, q^{\prime}(n)\right\} \cap R\right| \geqslant 2 n+1$;

2) $n \neq m \Longrightarrow\left\{h\left(q^{\prime}(n)\right), \ldots, q^{\prime}(n)\right\} \cap\left\{h\left(q^{\prime}(m)\right), \ldots, q^{\prime}(m)\right\}=\varnothing$.

По условию леммы такая функция $q^{\prime}$, очевидно, существует. Теперь определим множество $L$ и функцию $q$ так: $R(q(n))=\min \left(R \cap\left\{h\left(q^{\prime}(n)\right), \ldots, q^{\prime}(n)\right\}\right), L=\{R(q(n)) \mid n \in \mathbb{N}\}$. Ясно, что $L, R \backslash L$ - бесконечные рекурсивные множества. Рассмотрим множества $R_{-}=R \backslash L, \bar{R}_{+}=\bar{R} \cup L$ и определим функции $e_{+}: \bar{R} \rightarrow \bar{R}_{+}, e_{-}: R \rightarrow R_{-}$следующими равенствами: $e_{+}(\bar{R}(n))=\bar{R}_{+}(n), e_{-}(R(n))=R_{-}(n)$ для всех $n \in \mathbb{N}$. Обе $e_{+}$и $e_{-}-$peкурсивные функции на их областях определения. Кроме того, если определить $\alpha(P, n)$ как число элементов множества $L$, которое не превышает $P(n)(P$ - бесконечное множество), то

$$
e_{+}(\bar{R}(n)) \leqslant \bar{R}(n-\alpha(\bar{R}, n)) \leqslant \bar{R}(n)-\alpha(\bar{R}, n) .
$$

Также $e_{-}(R(n)) \leqslant R(n+\alpha(R, n))$.

Получаем $\bar{R} \cap A \leqslant_{m}^{H} e^{+}(\bar{R} \cap A)$, где если $n \in \bar{R}$, то $H(n)=e_{+}(n)$, иначе $H(n)=a$ для некоторого фиксированного элемента $R$. Таким образом, $H(\bar{R}(n)) \leqslant \bar{R}(n)-\alpha(\bar{R}, n)$.

С другой стороны, мы можем перестроить сводящее множество $W_{z}$, определяя сведение $A \cap R \leqslant_{(I-h)}^{z} X$ так, что $D_{u} \cup D_{v} \subseteq R$ для любой $\langle x, i, u, v\rangle \in W_{z}$. Действуя таким

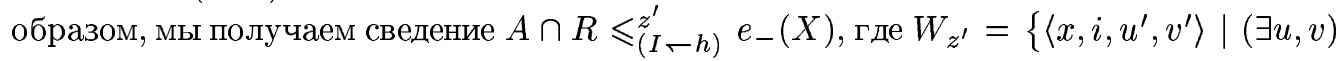
$\left(D_{u^{\prime}}=e_{-}\left(D_{u}\right) \& D_{v^{\prime}}=e_{-}\left(D_{v}\right) \&\langle x, i, u, v\rangle \in W_{z} \&\left(x_{0} \leqslant x \Longrightarrow \max \left(D_{u} \cup D_{v}\right) \leqslant\right.\right.$ $I(x)-h(x))\}$. Давайте оценим $\max \left(D_{u^{\prime}} \cup D_{v^{\prime}}\right)$, когда $\left\langle x, i, u^{\prime}, v^{\prime}\right\rangle \in W_{z^{\prime}}$ для данных $x, i$. Если $R(n) \leqslant I(x)-h(x)$, то $\left|R_{-} \cap\{R(n)+1, \ldots, x\}\right| \geqslant 2 \alpha\left(R_{-}, n\right)$. Тогда $R\left(n+2 \alpha\left(R_{-}, n\right) \leqslant x\right.$. Отсюда и из $e_{-}(R(n)) \leqslant R\left(n+\alpha\left(R_{-}, n\right)\right)$ следует, что $e_{-}(R(n)) \leqslant R\left(n+\alpha\left(R_{-}, n\right)\right) \leqslant x-\alpha\left(R_{-}, n\right)$.

Наконец, $A \leqslant\left(I-h^{\prime}\right) e_{+}(A) \cup e_{-}(X)$, где $h^{\prime}(n)=\max (\{\alpha(\bar{R}, k) \mid R(k) \leqslant n\} \cup\{\alpha(R, s) \mid$ $R(s) \leqslant n\})$.

Напомним, что множество назьвается иммунньг, если оно невключает никакого бесконечного рекурсивно перечислимого подмножества.

Теорема 1. Если $A$-рекурсивно несжимаемое множество, то как $A$, так и $\bar{A}$ являются иммунными. Обращение этого утверэсдения ложно.

ДокАЗАТЕЛЬСтво. Первое утверждение вытекает непосредственно из предыдущих лемм. Достаточно заметить, что для каждого рекурсивного множества $R \subseteq A$ и любого множества $X$ имеем $R \leqslant 。 \quad X$, где $o(x)=0$ для всех $x \in \mathbb{N}$.

Теперь построим по шагам пример такого множества $A$, что противоречит обращению первого утверждения леммы. Пусть $R$ обозначает множество $\left\{2^{2^{n}} \mid n \in \mathbb{N}\right\}$ и пусть уже определен сегмент $\mathscr{C}_{A}^{2 n}$ характеристической функции $\mathscr{C}_{A}$ множества $A$. Длину сегмента $\mathscr{C}_{A}^{2 n}$ обозначим через $d_{2 n}$.

Шаг $2 n$. Если $W_{n} \cap R$ - конечное множество, то положим $d_{2 n+1}=d_{2 n}, \mathscr{C}_{A}^{2 n+1}=\mathscr{C}_{A}^{2 n}$ и переходим к следующему шагу. Иначе, находим первые два элемента $x, y \in W_{n} \cap R$ такие, что $d_{2 n}<x<y$, и полагаем $\mathscr{C}^{2 n+1}(y)=1, d_{2 n+1}=y, \mathscr{C}_{A}^{2 n+1}(z)=0$ для всех $d_{2 n}<z<d_{2 n+1}$. Переходим к следующему шагу. 
Шаг $2 n+1$. Если $W_{n} \cap \bar{R}$ является конечным множеством, то полагаем $d_{2 n+2}=d_{2 n+1}$, $\mathscr{C}_{A}^{2 n+2}=\mathscr{C}_{A}^{2 n+1}$ и переходим к следующему шагу. В противном случае найдем первые два элемента $x, y \in W_{n} \cap \bar{R}$ такие, что $d_{2 n+1}<x<y$ и положим $\mathscr{C}^{2 n+2}(y)=1$, $d_{2 n+2}=y, \mathscr{C}_{A}^{2 n+2}(z)=0$ для всех $d_{2 n+1}<z<d_{2 n+2}$. Переходим к следующему шагу.

Положим $\mathscr{C}_{A}=\bigcup_{n} \mathscr{C}_{A}^{n}$. Ясно, что оба множества $A$ и $\bar{A}$ являются иммунными. Теперь определим $X=\{\sqrt{x} \mid x \in R \cap A\}$. Имеем $X \subseteq R$ и $R \cap A \leqslant{ }_{h} X$, где $h=\lambda n \cdot[\sqrt{n}]$. Таким образом, $A$ есть РСМ по лемме 2.

Следующее утверждение является вариантом известной теоремы Барздиня, а доказательство - модификацией соответствующей конструкции [4].

ТЕорема 2. Для любих рекурсивно перечислимого множества (РПМ) $A и \varepsilon>0$ существует множество $B$, для которого $A \leqslant_{\left[\log _{2}^{1+\varepsilon}\right]} B$.

ДокАЗАТЕЛьство. Достаточно рассмотреть нерекурсивное РПМ $A$. Пусть рекурсивная 1-1-функция $f$ перечисляет $A$. Фиксируем некоторые числа $k, n_{0} \in \mathbb{N}$, зависящие от $\varepsilon$. Определенно мы можем включить информацию о начальном сегменте $\left.\mathscr{C}_{A}\right|_{0} ^{2 \exp n_{0}}$ в программу $z$, сводя $A$ к $B$.

Определим $t_{n}=\max \left\{x \mid f(x)<2 \exp n^{k}\right\}, n>n_{0}$, и построим $\mathscr{C}_{B}$ как катенацию 01-кодов чисел $f\left(t_{n}\right), n=n_{0}+1, n_{0}+2, \ldots$. Здесь 01-код $f\left(t_{n}\right)$ есть слово $01 \nu(1) \ldots 1 \nu(i) \ldots 1 \nu(m) 0$, где $\nu(1) \ldots \nu(m)$ - бинарная запись $f\left(t_{n}\right)$. 01-код $f\left(t_{n}\right)$ назовем $\left(n ; n_{0}\right)$-блоком $\mathscr{C}_{B}$. Очевидно, что длины блоков не убывают.

Чтобы вычислить $\mathscr{C}_{A}(x)$ при $x>2 \exp n_{0}^{k}$, определим сначала $n(x)=\mu v\left(x<2 \exp v^{k}\right)$. Затем раскодируем $f\left(t_{n(x)}\right)$, выделяя $\left(n(x) ; n_{0}\right)$-й блок в $\mathscr{C}_{B}$. Затем мы вычисляем $f(0), \ldots, f(p)$ до тех пор, пока не обнаружится $f(p)=f\left(t_{n(x)}\right)$. Если $x \in\{f(0), \ldots, f(p)\}$, полагаем $\mathscr{C}_{A}(x)=1$, в противном случае $\mathscr{C}_{A}(x)=0$.

Так как длина $\left(n(x) ; n_{0}\right)$-блока последовательности $\mathscr{C}_{B}$ не превьшает $2 n^{k}(x)+2$ и $n(x) \leqslant \log _{2}^{1 / k}(x+1)$, то длина использованного начального сегмента последовательности $\mathscr{C}_{B}$ не больше чем $\sum_{i=1}^{n(x)} 2\left(i^{k}+1\right)$, т.е. не больше чем $3\left[\log _{2}^{1 / k} x\right]^{k+1}$ почти всюду. Учитьвая произвольность $k$, можно избавиться от множителя 3 . Это и дает объявленную оценку.

Иногда естественно представлять данное РПМ в виде объединения рекурсивных множеств, являющихся "рекурсивными вариациями" элементов некоторого рекурсивно перечислимого множества. Более строго, мы скажем, что РПМ $A$ имеет кусочно рекурсивное $f$-растянутое представление для рекурсивной функции $f \in \mathscr{T}^{+}$, если существуют рекурсивная одноместная функция $r$, частично рекурсивная двухместная $\varphi$ и РПМ $W_{e}$ такие, что

i) $\left(\forall x \in f\left(W_{e}\right)\right)\left(\left(W_{r(x)}\right.\right.$ рекурсивно) \& ( $x$ есть минимальньй элемент $\left.\left.W_{r(x)}\right)\right)$;

ii) $\left(\forall x \in f\left(W_{e}\right)\right) \forall y\left(\mathscr{C}_{W_{r(x)}}(y)=\varphi(x, y)\right)$;

iii) $A=\bigcup_{x \in f\left(W_{e}\right)} W_{r(x)}$.

Далее, если $h \in \mathscr{T}^{+}$, то $(h)^{-1}$ обозначает функцию $\lambda y \cdot(\mu x(h(x)>y))$.

СлЕдСТВИЕ 1. Если $A$ имеет $f$-растянутое кусочно рекурсивное представление $u \varepsilon>0$, то существует множсество $B$ такое, что $A \leqslant_{\log _{2}^{1+\varepsilon} \circ(f)^{-1}} B$.

ДокАЗАТЕЛЬСТво. Из условия следует, что для определения верно ли $x \in A$, нам достаточно определить, какие числа из множества $\left\{0, \ldots,(f)^{-1}(x)\right\}$ принадлежат множеству $W_{e}$. Теперь используем теорему 2. 
До сих пор мы занимались бинарными 01-массивами, содержашими рекурсивно перечислимое множество единиц. Можно свести $k$-значньй массив $F: \mathbb{N} \rightarrow\{0, \ldots, k-1\}$, когда $\{x \mid F(x)>0\}$ рекурсивно перечислимо, к рассмотренному вьше случаю путем следующего кодирования. Пусть $w(i)$ будет бинарньм кодом числа $i \in\{0, \ldots, k-1\}$ и $\|w(i)\|=z(k)$, где $z(k)=\mu z\left(\log _{2} k \leqslant z\right)$. Тогда мы представляем массив $F$ множеством $A$ с $\left.\mathscr{C}_{A}\right|_{0} ^{(n+1) z(k)-1}=w(F(0)) w(F(1)) \ldots w(F(n)), n \in \mathbb{N}$.

Если $\{x \mid F(x) \neq 0\}$ рекурсивно перечислимо, то $A$ также РПМ. Поэтому в соответствии с теоремой 2 мы можем сжать $\left.\mathscr{C}_{A}\right|_{0} ^{x}$ в $\log _{2}^{1+\varepsilon}(x)$ раз, получая множество $B$. Наконец, раскодируя $B$, мы получаем $k$-значньй массив $G$, сжимаюший $F$ в $\log _{2}^{1+\varepsilon}$ раз.

Такое же обобщение верно и для следствия 1.

Если $w$ является 01-словом, то $K(w)$ обозначает его сложность по Колмогорову [5]. Мы пишем $f \preccurlyeq h$, если $\exists c \forall x(f(x) \leqslant h(x)+c)$.

Лемма 3. Если $A \leqslant{ }_{h} B, \operatorname{mo} K\left(\left.\mathscr{C}_{A}\right|_{0} ^{n}\right) \preccurlyeq h(n), n \in \mathbb{N}$.

ДокАЗАТЕЛЬСтво. Пусть $A \leqslant{ }_{h}^{z} B$ для подходящего числа $z$. Тогда программу для вычисления $\left.\mathscr{C}_{A}\right|_{0} ^{n}$ можно составить из простой управляющей программы $P^{\prime}$, программы $P^{\prime \prime}(n)$, вычисляющей $\left.\mathscr{C}_{B}\right|_{0} ^{h(n)}$, и программы $P_{z}$ для перечисления $W_{z}$. Программа $P^{\prime}$ находит четверки $\langle x, i, u, v\rangle \in W_{z}$ с помощью $P_{z}$ и строит $\left.\mathscr{C}_{A}\right|_{0} ^{n}$, проверяя $D_{u} \subseteq B \& D_{v} \subseteq \bar{B}$ с помошью программы $P^{\prime \prime}(n)$. Учитывая $\left\|P^{\prime}\right\|+\left\|P_{z}\right\|=$ const \& $K\left(\left.\mathscr{C}_{B}\right|_{0} ^{h(n)}\right) \preccurlyeq h(n)$, мы получаем требуемую оценку.

Обозначим через $\mathscr{T}^{+}(C)$ класс всех рекурсивных в $C$ тотальных неубывающих функ-

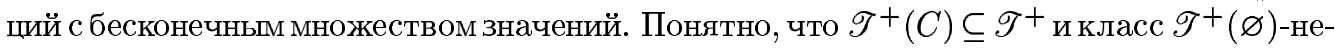
сжимаемых множеств совпадает с классом рекурсивно несжимаемьх множеств.

Теорема 3. Если $\underline{C}^{\prime} \leqslant d_{T}(B)$, то существует $\mathscr{T}^{+}(C)$-несжимаемое множесmво $A \in d_{T}(B)$.

ДокАЗАТЕЛЬСТво. Пусть $m \geqslant 2$ - фиксированное натуральное число и $p(x), x \in \mathbb{N}$, - программа, которая для произвольного слова удаляет его начало длины $x$, если это возможно, и выдает пустое слово $\Lambda$ иначе. Ясно, что $p(x)$ можно выбрать таким образом, что для некоторого числа $x_{0}$ верно $\|p(x)\| \leqslant x$, как только $x \geqslant x_{0}$.

Теперь по определению $p$ имеем $K(\sigma \tau) \geqslant K(\tau)-(\|p(\|\sigma\|)\|+c)$ для некоторой постоянной $c \in \mathbb{N}$ и любых слов $\sigma, \tau$. Фиксируем эту постоянную $c$.

Опираясь на это неравенство, лемму 3 и оракул $B$, можно диагональной процедурой построить $\mathscr{T}^{+}(C)$-несжимаемое множество $A$. Строим характеристическую функцию искомого множества как бесконечную 01-последовательность $\sigma$ по шагам.

Положим $\sigma_{0}=\Lambda$. Пусть после $(n-1)$-го шага мы имеем начальный сегмент $\sigma_{n}$ последовательности $\sigma$. Зададим $\sigma_{n+1}$ как $\sigma_{n} \psi_{n} \tau$, где $\psi_{n}=01^{\left\|\sigma_{n}\right\|+1} 0\left(\mathscr{C}_{B}(n)\right)$ и конечная последовательность $\tau$ выбрана следующим образом.

Пусть $f(n)=\left\|\sigma_{n} \psi_{n}\right\|$. С оракулом $B$ проверим условие $\mathfrak{A}:$ существует такое $x \in \mathbb{N}$, что

1) $\min \left\{x, \varphi_{n}^{C}(x+f(n))\right\}>2 f(n)+c+m$;

2) $(\forall y \leqslant x+f(n))(\forall z \leqslant y)\left(y \in W_{n}^{C} \& \varphi_{n}^{C}(z) \leqslant \varphi_{n}^{C}(y)\right)$.

Если это условие удовлетворено, то мы выберем первое такое число $x$. (Это возможно, так как множество таких $x$ перечислимо с оракулом $C$, а по условию теоремы всякое перечислимое в $C$ множество рекурсивно относительно $B$. В частности, проблема непустоты перечислимого множества разрешима с этим оракулом.) 
После этого мы имеем возможность выбрать с оракулом $B$ первое слово $\tau$ длины $x$, удовлетворяющее условию $K(\tau)>\|\tau\|-m$. Действительно, как хорошо известно [5], количество слов $\tau$ длины $x$ и сложности $K(\tau)$, которая больше чем $x-m$, больше чем $2^{x}\left(1-2^{1-m}\right)$. А так как любое перечислимое множество сводимо к $B$, можно вычислять $K(\tau)$ с оракулом $B$.

Если условие $\mathfrak{A}$ не удовлетворено, мы полагаем $\tau=\Lambda$.

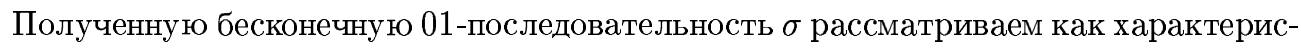
тическую функцию множества $A$.

Покажем $A \in d_{T}(B)$. Действительно, $A \leqslant_{T} B$, так как $A$ построено с оракулом $B$. А благодаря возможности находить подслова $\psi_{n}, n \in \mathbb{N}$, в $\mathscr{C}_{A}$ мы можем вычислить $\mathscr{C}_{B}$.

Из построения вытекает, что $A \leqslant\left(I_{\left.-\varphi_{l}^{C}\right)} X\right.$ ложно для каждой $\varphi_{l}^{C} \in \mathscr{T}^{+}$и каждого $X \subseteq \mathbb{N}$. Действительно, если $\varphi_{l}^{C} \in \mathscr{T}^{+}$, тогда по лемме 3 существует такое $k$, что $(\forall x \geqslant k)\left(K\left(\left.\mathscr{C}_{A}\right|_{0} ^{x} \leqslant x+k-\varphi_{l}^{C}(x)\right)\right.$. Перейдем к произвольному индексу $n$ функции $\varphi_{n}^{C}(x)=\varphi_{l}^{C}(x)-k$. На шаге $n$ слово $\tau$ было выбрано так, что

$$
\begin{aligned}
K\left(\sigma_{n} \psi_{n} \tau\right) & \geqslant K(\tau)-\left(\left\|p\left(\left\|\sigma_{n} \psi_{n}\right\|\right)\right\|+c\right)>\|\tau\|-\left(m+c+\left\|\sigma_{n} \psi_{n}\right\|\right) \\
& =\left\|\sigma_{n} \psi_{n}\right\|+\|\tau\|-\left(m+c+2\left\|\sigma_{n} \psi_{n}\right\|\right) \geqslant\left\|\sigma_{n} \psi_{n} \tau\right\|-\varphi_{n}^{C}\left(\left\|\sigma_{n} \psi_{n} \tau\right\|\right)
\end{aligned}
$$

так как $\varphi_{n}^{C}\left(\left\|\sigma_{n} \psi_{n} \tau\right\|\right) \geqslant 2\left\|\sigma_{n} \psi_{n}\right\|+m+c$ в соответствии с условием $\mathfrak{A}$. Рассуждение завершает стандартное соображение о бесконечности множества индексов любой функции из $\mathscr{T}^{+}$.

СлЕДСТВИЕ 2. В степени всякого множества $B$, для которого $\varnothing^{\prime} \leqslant_{T} B$, найдется рекурсивно несжимаемое множество.

Было бы интересно описать все степени рекурсивно несжимаемых множеств.

Рассмотрим естественную задачу существования и описания степеней, которые включают $\mathscr{T}^{+}$-несжимаемые множества, иначе говоря, - абсолютно несжимаемые множества. Обозначим класс абсолютно несжимаемых множеств через ANC.

ТЕОрема 4. Почти все (для обычной меры в пространстве бесконечных 01-последовательностей) множества $\mathscr{T}^{+}{ }_{-}$несжимаемы. Существует такое $\mathscr{T}^{+}{ }_{-} e_{-}$ сжимаемое множество $A$, что $\underline{A}^{\prime}=\underline{\varnothing}^{\prime \prime}$.

ДокАзАТЕЛЬСтво. Мартин-Лёф доказал [6], что для почти всех бесконечных 01-последовательностей $w$ сушествуют такие натуральные $m$, что $\exists^{\infty} n\left(K\left(\left.w\right|_{0} ^{n}\right)>n-m\right)$. Отсюда и из леммы 3 вытекает, что почти все множества натуральных чисел $\mathscr{T}^{+}{ }_{- \text {несжи- }}$ маемы.

Чтобы получить второе утверждение, сначала построим непустой П $\frac{\varnothing^{\prime}}{1}$-класс 01-последовательностей, каждая их которых представляет абсолютно несжимаемое множество. Тогда требуемое будет следовать из релятивизованного результата ДжокушаCoара (см. [7, теорема 1]).

Назовем $(e, m)$-правильной всякую такую 01-последовательность $\sigma$, что для неенайдется множество $\left\{\sigma_{1}, \ldots, \sigma_{e}\right\}$ различных начальных ее отрезков с условием $K\left(\sigma_{i}\right) \geqslant$ $\left\|\sigma_{i}\right\|-m$.

Определим предикат $\mathfrak{R}_{m}(n, k, \alpha) \Longleftrightarrow$ (доля $(k, m)$-правильных 01-последовательностей среди всех 01-последовательностей длины $n$ не меньше $\alpha$ ).

Теперь построим рекурсией относительно $\varnothing^{\prime}$ функции $\psi_{m}(n), \varepsilon_{m}(n)$. 
Значение $\varepsilon_{m}(n+1)$ равно максимальному числу $\alpha$ среди чисел $\varepsilon_{m}(n), f\left(\psi_{m}(n)\right)$, для которого $\mathfrak{R}_{m}\left(n+1, \psi_{m}(n), \alpha\right)=1$. Здесь $f$ - указанная далее вычислимая функция.

Значение $\psi_{m}(n+1)$ равно $\psi_{m}(n)$, если $\varepsilon_{m}(n+1)<f\left(\psi_{m}(n)\right)$, а иначе равно максимальному числу $a \in\left\{\psi_{m}(n), \psi_{m}(n)+1\right\}$, для которого $\mathfrak{R}_{m}\left(n+1, a, \varepsilon_{m}(n+1)\right)=1$.

Положим $f(k)=1-2^{-k-2}, \psi_{m}(0)=0, \varepsilon_{m}(0)=1 / 2$.

Очевидно, $\psi_{m}, \varepsilon_{m}$ - тотальные неубьвающие функции, вычислимые относительно $\underline{\varnothing}^{\prime}$ с бесконечными областями значений. Последнее вытекает из вышеупомянутого результата Мартин-Лёфа. Действительно, для любых $p \in \mathbb{N}, e \in[0,1)$ найдется такое число $n_{p, e} \in \mathbb{N}$, что верно утверждение $\mathfrak{R}_{m}\left(n_{p, e}, p, e\right)$.

Объявленњый выше П $\frac{\varnothing^{\prime}}{1}$-класс определяется как

$\left\{X \mid \forall n\right.$ (начальньй сегмент $\mathscr{C}_{X}$ длины $n$ является $\left(\psi_{m}(n), m\right)$-правильной $\left.)\right\}$.

Этот класс непуст, так как $\sum_{n=2}^{\infty} 2^{n}<1$.

Существуют ли степени абсолютно несжимаемых множеств с более низкой верхней оценкой неразрешимости, чем в этой теореме?

Определим сводимость $\leqslant_{T}+$ следующим образом:

$$
A \leqslant_{T^{+}} B \Longleftrightarrow\left(\exists f \in \mathscr{T}^{+}\right)\left(A \leqslant_{(I-f)} B \vee A \leqslant_{I} B\right)
$$

Это отношение сводимости, так как его рефлексивность и транзитивность очевидны, как и включение $\leqslant_{T^{+}} \subseteq \leqslant_{T}$. Заметим сразу же, что $\leqslant_{T^{+}}$-степень произвольного нерекурсивного множества $A$ состоит из множеств, сводящихся к $A$ с точной границей $I$. Из полученных вьше, а также известных результатов вытекает, что частичньй порядок $L_{T^{+}}$, индуцированный сводимостью $\leqslant_{T^{+}}$на множествах ее степеней неразрешимости соответственно, имеет необычные свойства. Например:

Теорема 5. 1) Мнохсество максимальных в $L_{T^{+}}$степеней несчетно.

2) Не существует минимальных нерекурсивных степеней в $L_{T^{+}}$.

$3) \leqslant_{T^{+}}$-степень креативного мнохества $A$ не максимальна в $d_{T}(A)$.

4) Существуют пары мнохеств, тьюринговы степени которых не превосходят $\underline{\varnothing}^{\prime}$, не имеющие точной верхней грани в $L_{T^{+}}$.

ДокАЗАтЕльство. Утверждение 1) есть следствие того, что всякое абсолютно несжимаемое множество имеет максимальную степень и каждая степень не более чем счетна.

Чтобы доказать утверждение 2) рассмотрим произвольное нерекурсивное множество $A$. Множество $2 A \rightleftharpoons\{2 a \mid a \in A\}$ также нерекурсивно. Ясно, что $2 A \leqslant_{I-\lambda x \text {.[x/2] }} B$, но $A \nless T^{+} 2 A$ из-за нерекурсивности $A$.

Утверждение 3) следует из леммы 2. Действительно, всякое РПМ рекурсивно сжимаемо.

4) Возьмем рекурсивно несжимаемое множество $A$ и рассмотрим его как сочленение $B \oplus C$, где $B \rightleftharpoons\{a \mid 2 a \in A\}, C \rightleftharpoons\{a \mid 2 a+1 \in A\}$. Они тоже рекурсивно несжимаемы в силу леммы 2 . Если бы они имели верхнюю грань в нашей $L_{T^{+}}$, то их сочленение также очевидно было бы рекурсивно сжимаемо.

Ниже $\mathrm{KR}_{B}(n)$ обозначает $\mathrm{KR}\left(\mathscr{C}_{B}(0) \ldots \mathscr{C}_{B}(n)\right)$, где $\mathrm{KR}(\cdot)$ - сложность по Ловеланду-Маркову [8]. 
Teopema 6. Пусть A ecmb РПМ.

Тогда $(A$ является $T$-полным $) \Longleftrightarrow \exists B\left(\exists t \in \mathscr{T}^{+}\right)\left(B \notin \unrhd \& B \leqslant_{T^{+}} A \& t \leqslant_{T} A\right.$ $\left.\& t \preccurlyeq \mathrm{KR}_{B}\right)$.

ДокаЗАТЕЛЬСтво. Если $B \leqslant h A, h \leqslant I$, то (см. [1]) $\mathrm{KR}_{B}(n) \leqslant c+\mathrm{KR}_{A}(h(n))$, т.е. $\mathrm{KR}_{B}(n)-c \leqslant \mathrm{KR}_{A}(n)$ для некоторого $c \in \mathbb{N}$ и почти всех $n \in \mathbb{N}$. Следовательно, существует такая функция $f \in \mathscr{T}^{+}$, что $f \leqslant T$ $A \& f \leqslant \mathrm{KR}_{A}$. Как показано в [1], $A$ является полным множеством.

Обратно, если $A$ полно и для некоторого множества $B$ верно $B \leqslant \mathscr{T}^{+} A$, то $\mathrm{KR}_{B} \leqslant T_{T} A$. Выберем $B=\{2 x \mid x \in A\}$. Тогда $B \leqslant_{\lambda x}$.([x/2]+1) $A$ очевидно. Поэтому в качестве $t$ можно выбрать $\mathrm{KR}_{B}$.

СледСТВИЕ 3. РПМ $A$ не является полным тогда и только тогда, когда $B \Varangle w t t$ $A \vee \mathrm{KR}_{B} \Varangle_{T} A$ для каждого нерекурсивного множества $B$.

ДоказАтельСтво. Пусть $A$ РПМ, не являющееся полным, и $B \leqslant w t t A$. Тогда существует рекурсивная 1-1-функция $g \in \mathscr{T}^{+}$такая, что $B \leqslant g$. Имеем

$$
y \in g(B) \Longleftrightarrow y \in \operatorname{Val} g \& g^{-1}(y) \in B
$$

Следовательно, $g(B) \leqslant{ }_{I} A$. Кроме того, ясно, что $\mathrm{KR}_{B} \preccurlyeq \mathrm{KR}_{g(B)} \circ g$. Тогда существует такое число $c$, что $\mathrm{KR}_{B}(n-\mu k(g(n-k) \leqslant n))-c \leqslant \mathrm{KR}_{g(B)}(n)$ для каждого $n \in \mathbb{N}$. Поэтому из $\mathrm{KR}_{B} \leqslant T A$ вытекает существование рекурсивной в $A$ функции, которая минорирует $\mathrm{KR}_{g(B)}$. Это противоречит теореме 4 .

Обратно, если $A$ полное РПМ, то $A \leqslant w t t A$ и $\mathrm{KR}_{A} \leqslant T A$.

\section{СПИСОК ЦИТИРОВАННОЙ ЛИТЕРАТУРЫ}

[1] Булитко В. К. Субтьюринговы сводимости ограниченной сложности // Изв. вузов. Матем. 1992. №1. C. 27-37.

[2] Соловьёв В. Д. Структура распределения информации в бесконечной последовательности // Дискретная матем. 1996. Т. 8. № 2. С. 97-107.

[3] Роджерс Х. Теория рекурсивных функций и эффективная вычислимость. М.: Мир, 1972.

[4] Барздинь Я. М. Сложность программ, распознающих принадлежность натуральных чисел, не превьшающих $n$, рекурсивно перечислимому множеству // Докл. АН СССР. 1968. Т. 182. №6. C. $1249-1252$.

[5] Колмогоров А. Н. Три подхода к определению понятия "количество информации" // Проблемы передачи информации. 1965. Т. 1. №1. С. 3-11.

[6] Martin-Löf P. Complexity oscillations in infinite binary sequences // Z. Wahrscheinlichkeitsteorie Verw. Geb. 1971. V. 19. № 3. P. 225-230.

[7] Jockush C. G., Soare R. I. $\Pi_{1}^{0}$-classes and degrees of theories // Trans. Amer. Math. Soc. 1972. V. 173. P. 33-56.

[8] Loveland D.W. A variant of the Kolmogorov concept of complexity // Inform. and Control (Shenyang). 1969. V. 15. P. 510-526.

Одесский государственный университет

Поступило

E-mail: booly@te.net.ua 14.08 .96

Исправленный вариант 